\title{
Kisspeptin and spexin: lipid profile and sperm parameters in infertile and fertile male subjects
}

\author{
Mohummad Hassan Raza ${ }^{a}$, Mussarat Ashraf ${ }^{b}$, Sher Khan ${ }^{b}$, Nida Farooqui ${ }^{c}$, Arfa Azhard, Rehana Rehman \\ ${ }^{a}$ Medical Student, Department of Biological \& Biomedical Sciences, Aga Khan University Karachi. \\ ${ }^{b}$ Senior Technologist, Department of Biological \& Biomedical Sciences, Aga Khan University Karachi.

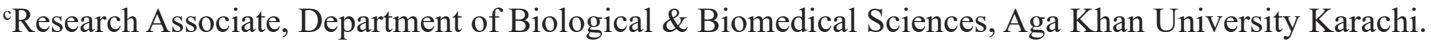 \\ ${ }^{\mathrm{d} S e n i o r ~ I n s t r u c t o r, ~ D e p a r t m e n t ~ o f ~ B i o l o g i c a l ~ \& ~ B i o m e d i c a l ~ S c i e n c e s, ~ A g a ~ K h a n ~ U n i v e r s i t y ~ K a r a c h i . ~}$ \\ eAssociate Professor, Department of Biological \& Biomedical Science, Aga Khan University Karachi. \\ *Corresponding author:drrehana7@,gmail.com
}

\section{ABSTRACT}

BACKGROUND \& OBJECTIVE: Kisspeptin (KP) and spexin (SPX) are neuropeptides that play a substantial role in metabolism and sexual function. Our objective is to explore the association of spexin and kisspeptin with sperm parameters and lipid profile in the infertile male population.

METHODOLOGY: The cross-sectional study was conducted from March 2017 till July 2018 in Aga Khan University Hospital, Karachi, Pakistan, after acquiring ethical approval (4813-BBS-ERC-17). Convenient sampling was applied to recruit 44 normozoospermic subjects as controls and 44 patients with abnormal sperm parameters". Serum levels of kisspeptin and spexin were measured by enzyme linked immunosorbent assay and Cobas c111 analyzer was used for estimation of high and low density-lipoprotein, triglyceride and serum cholesterol levels. Mann-Whitney U tests and spearman's rank correlation test were used to analyze quantitative variables, and data was presented by means, standard error (SE) and correlations coefficient, with a statistical significance set at $\mathrm{p} \leq 0.05$.

RESULTS: The mean kisspeptin values of 'altered sperm parameters' males $13.23 \pm 3.34$ were lower than normal sperm parameters $31.89 \pm 3.25(\mathrm{p}<0.001)$. The mean spexin values of altered sperm parameters $383.57 \pm 21.09$ were less than compared to normal sperm parameters $502.76 \pm 19.77(\mathrm{p}<0.001)$. Correlation of KP and SPX levels was $(\mathrm{r}-\mathrm{value}=0.332, \mathrm{p}=$ 0.002). There was no significant correlation was found between KP and SPX with lipid profile parameters.

CONCLUSION: Spexin and kisspeptin levels observed were higher in male subjects with normal sperm parameters and had a significant positive correlation with each other. There was no impact of spexin on lipid profile and obesity.

KEYWORDS: Kisspeptin, Spexin, Lipid Profile, Obesity, Infertility.

\section{INTRODUCTION}

Kisspeptin (KP) and spexin (SPX) are closely related neuropeptides that play a substantial role in metabolism, body weight and sexual function. Genes for both peptides lie close to one another, and both are members of the same gene family [(SPX/GAL/KISS $)]^{[1]}$.

$\mathrm{KP}$ is encoded by the kisspeptin 1 gene (KISS1), which is prominently involved in fertility, primarily through the modulation of the hypothalamo pituitary gonadal (HPG) axis. The effects of KP are highly mediated when it interacts with its kisspeptin 1 receptor (KISS1R) that is responsible for secreting gonadotropin releasing hormone $(\mathrm{GnRH})^{[2]}$. Therefore, dysfunction in KP or KISS1R may lead to infertility, through the abnormal secretion of GnRH. Abnormalities in KP and KISS1R are largely caused through mutations, for example Rehman et al, studied that mutations and single nucleotide polymorphisms in exons 2 and 3 of KISS1 gene lead to decreased levels of KP and subsequently infertility ${ }^{[3]}$.
In addition to that KP also plays a part in the mediation of insulin resistance and obesity as Kołodziejski et al ${ }^{[4]}$ observed that obesity is linked with lower levels of KP, and a negative correlation exists between KP and body mass index (BMI) and KP and insulin resistance.

In 2014 Zhou et al. conducted a study on post pubertal rats, with the objective of exploring the association of obesity with ovulatory disorders on the Kiss1 system in the ovaries. In different studies, it was found that the rats had been fed with a high fat diet, and had subsequently developed obesity, exhibited on average lowers levels KP as well as decreased ovulatory capacity ${ }^{[5]}$. In one of our studies, we have concluded that Kisspeptinincreases the production of reproductive hormones $\mathrm{FSH}, \mathrm{LH}$ and testosterone by its effect on hypothalamic pituitary gonadal axis [3]. Literature suggests a potential link between obesity and infertility explained through the effect of adipocytokines on reproductive functions and fertility outcomes ${ }^{[6]}$. Neuropeptides take part in number of brain activities which take part in manifestation of higher mental functions ${ }^{[7]}$.

Raza MH, Ashraf M, Khan S, Farooqui N, Azhar A, Rehman R. Kisspeptin and Spexin: Lipid profile and sperm parameters in infertile and fertile male subjects. Journal of University Medical \& Dental College. 2021;12(4):235-239. 


\section{Kp and spx: a pilot study on male subjects}

SPX, also called neuropeptide Q (NPQ), is a novel peptide, only having being discovered in 2007, consisting of 14 amino acids. SPX itself binds to 2 receptors, galanin receptor 2 and galanin receptor 3 , and it has been found to be expressed in various tissues and organs like heart, lungs, ovaries, testis and brain ${ }^{[1]}$. Its actual functions are still unknown; however, it is hypothesized that SPX may play a role in several physiological processes including weight loss and energy metabolism, uptake of fatty acids, glucose and lipid metabolism, movement in the gastrointestinal tract, renal and cardiovascular function as well as effects on the central nervous system ${ }^{[1]}$. Among the established roles of SPX, its role is more on weight loss and energy metabolism. It was found that gene coding for SPX (C12orf29) showed a 14-fold decrease in expression in the subcutaneous and omental adipocytes of obese individuals ${ }^{[8]}$. These previous findings have been corroborated by the fact Chengyuan et al. discovered that circulating levels of SPX negatively correlate with BMI without any correlation between SPX and cholesterol ${ }^{[9]}$. A negative correlation between SPX and (LDL and Triglycerides) has additionally been reported ${ }^{[10]}$. The above findings indicate that SPX has a marked effect on the lipid metabolism of an individual, with low serum SPX levels thought to be a potential biomarker for adult obesity [11].

Low levels of spexin were found to be related with childhood obesity ${ }^{[12]}$. Spexin is related with energy metabolism, feeding behavior and endocrine functions ${ }^{[13]}$. However, this may be species specific, as Zheng et al. showed that mutant zebrafish although fertile are still unable to produce SPX (SPX knockout) and no unusual finding was recorded in terms of maturation of gamete or onset of puberty in both males and females ${ }^{[14]}$. Nevertheless, SPX and its relationship with fertility in humans, still largely remains unknown.

Whereas literature is present on KP, there is a dearth of literature on SPX, with most studies involving SPX being restricted to animals. No study has been conducted, among the Pakistani population, which has assessed the levels of SPX. Furthermore, the link between the levels of these peptides (KP and SPX) and the lipid profile in fertile and infertile individuals is yet to be found in the Pakistani population. This indicates a significant gap in knowledge and results from this study may provide a possible link between these peptides, obesity and infertility in a South Asian context.

\section{METHODOLOGY}

Our study was cross-sectional, which was conducted from March 2017 till July 2018 in Aga Khan University Hospital, Karachi, Pakistan. The institute provided the institutional ethical review (4813-BBS-ERC-17). We included all subjects who approached the clinic during the study period, performed their semen analysis and categorized the subjects into normal and altered sperm parameters. Normal parameters had a total sperm count of (TC) $>39$ million per ejaculate, total sperm motility $>40 \%$, and normal morphology of $\geq 4 \%$. The subjects were considered to have abnormal sperm parameters if they fall in the category as defined by WHO criteria ${ }^{[15]}$ i.e.,) $\mathrm{TC}<39$ million sperms per ejaculate ii) less than $40 \%$ motility, and iii) less than $4 \%$ normal morphology. A convenient sampling technique was applied to recruit 88 male subjects between the ages of 25 and 55 years, out of which 44 (controls) had 'normal' sperm parameters (normozoospermic), and 44 had 'altered sperm parameters. Individuals with other causes of infertility, like cryptorchidism, testicular trauma, orchitis or testicular hypotrophy were excluded from the study. Subjects with liver/renal diseases, arthritis, malignancy, diabetes, epilepsy, endocrinal disorders, hypertension, tuberculosis, and those receiving steroids/drugs, testosterone or thyroxin replacement therapy are also excluded. After obtaining consent from all study participants to participate, a general physical examination was performed, and body mass index (BMI) was calculated. After collecting the blood samples from the subjects, serum was separated through centrifugation.

BMI of the study participants was categorized according to cutoffs for Asian considering overweight $(\geq 25 \mathrm{~kg}$ / $\mathrm{m}^{2}$ ) population with a high risk of type II diabetes and cardiovascular diseases ${ }^{[16]}$. The cutoffs used for body fat (BF\%) for normal weight was (12-22\%), overweight (22.1$27 \%)$ and obese $(>27.1 \%)^{[17]}$. Serum samples were used to measure kisspeptin levels by commercially available enzyme-linked immunosorbent assay (ELISA) Kit (Cat. No: 95611, Glory Bio Science, USA) with a detection range of $5 \mathrm{ng} / \mathrm{L}-180 \mathrm{ng} / \mathrm{L}$. The analytical sensitivity of the kit was $10.16 \mathrm{ng} / \mathrm{L}$, and intra- and inter-assay coefficient of variation was $<10 \%$ and $12 \%$ respectively. For detection of serum spexin levels commercially available ELISA kit (Cat. No: E3507Hu, Bioassay Technology Laboratory, China) was utilized with a detection range of $10 \mathrm{ng} / \mathrm{L}-4000 \mathrm{ng} / \mathrm{L}$. The analytical sensitivity of the assay was $4.95 \mathrm{ng} / \mathrm{L}$, and the intra- and inter assay coefficient of variation were $<8 \%$ and $<10 \%$, respectively, according to the manufacturers protocol. Serum levels of cholesterol, triglyceride, HDL and LDL were measured using Cobas c111 analyser (Roche Pakistan Ltd.).

Data was analysed using IBM SPSS Version 20. The quantitative variables were described by means and standard error of the mean (SE). The Mann Whitney $U$ test and Spearman's rank correlation test were used to compare and correlate them respectively. The significance of the results was set at $\mathrm{p} \leq 0.05$.

\section{RESULTS}

The demographic and biochemical parameters of 88 male subjects are given in table-I. Age in the 'altered sperm parameters' group was $34.45 \pm 1.09$ years (mean \pm SE), and it was significantly lower as compared to the subjects with normal sperm parameters $37.50 \pm 0.94 \quad(\mathrm{p}<0.001)$. The mean values of body fat between 'altered sperm parameters' and 'normal sperm parameters were not statistically significant. Data is presented as Mean $\pm \mathrm{SE}$ for all variables, compared by Mann Whitney U Test, $\mathrm{p}<0.05$ considered statistically significant. The mean KP values of 'altered sperm parameters' males $13.23 \pm 3.34$ (ng/L) was significantly lower than 'normal sperm parameters' 31.89 $\pm 3.25(\mathrm{p}<0.001)$. 


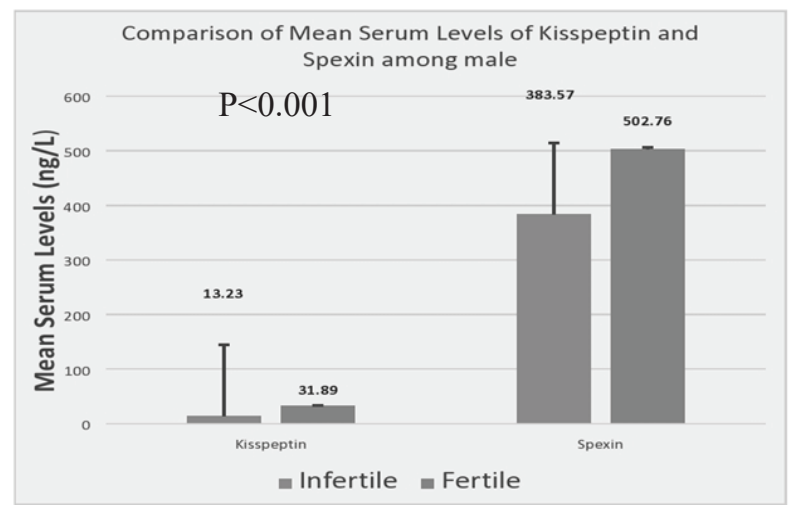

Figure-I: Comparison of mean serum levels of kisspeptin and spexin in subjects with normal and altered sperm parameters.

Table-I: Clinical and Biochemical data of Study Groups.

\begin{tabular}{cccc}
\hline Variables & $\begin{array}{c}\text { Altered Sperm } \\
\text { Parameters (Mean } \\
\pm \text { SE) } \mathbf{n}=\mathbf{4 4}\end{array}$ & $\begin{array}{c}\text { Normal } \\
\text { Sperm } \\
\text { Parameters } \\
\text { (Mean } \pm \text { SE) } \\
\mathbf{n}=\mathbf{4 4}\end{array}$ & p-value \\
\hline Age (years) & $34.45 \pm 1.09$ & $37.50 \pm 0.94$ & 0.013 \\
\hline $\begin{array}{c}\text { Body Mass Index } \\
\text { (kg/m2) }\end{array}$ & $25.89 \pm 0.47$ & $24.47 \pm 0.54$ & 0.087 \\
\hline $\begin{array}{c}\text { Body Fat (\%) } \\
\text { Kisspeptin (ng/L) }\end{array}$ & $33.6 \pm 0.69$ & $32.59 \pm 0.71$ & 0.494 \\
\hline $\begin{array}{c}\text { Spexin (ng/L) } \\
\text { Cholesterol (mg/dl) }\end{array}$ & $13.23 \pm 3.34$ & $31.89 \pm 3.25$ & 0.00 \\
\hline $\begin{array}{c}\text { Triglycerides (mg/ } \\
\text { dl) }\end{array}$ & $140.67 \pm 21.09$ & $502.76 \pm 19.77$ & 0.00 \\
\hline $\begin{array}{c}\text { High density lipid } \\
\text { protein C4 (mg/dl) }\end{array}$ & $34.59 \pm 1.32$ & $172.93 \pm 4.57$ & 0.640 \\
\hline $\begin{array}{c}\text { Low Density } \\
\text { Lipoprotein C3 } \\
\text { (mg/dl) }\end{array}$ & $102.95 \pm 4.50$ & $167.32 \pm 11.88$ & 0.246 \\
\hline $\begin{array}{c}\text { Sperm Count (mill/ } \\
\text { ml) }\end{array}$ & $30.86 \pm 2.30$ & $92.02 \pm 7.58$ & 0.00 \\
\hline $\begin{array}{c}\text { Motility (\%) } \\
\text { Morphology (\%) }\end{array}$ & $31.66 \pm 2.33$ & $68.23 \pm 2.05$ & 0.00 \\
\hline & $2.54 \pm 0.23$ & $4.05 \pm 0.24$ & 0.00 \\
\hline
\end{tabular}

The mean spexin values of 'altered sperm parameters' 383.57 \pm 21.09 (ng/L) was also considerably lesser as compared to 'normal sperm parameters'502.76 $\pm 19.77(\mathrm{p}<0.001)$.

In the lipid profile panel, the cholesterol (CHO2L), triglycerides (TRIG), high density lipid (HDLC4) and lowdensity lipid (LDLC3) levels were not significantly different between 'altered sperm parameters' and 'normal sperm parameters. Spearman's Rank Correlation applied, $\mathrm{p}<0.05$ considered as statistically significant. The Spearman's rank correlation of KP and SPX with lipid profile is shown in Table-II. A statistically significant positive correlation was detected between KP and SPX levels (r-value $=0.332$, $\mathrm{p}=0.002)$. Neither KP nor SPX showed any significant correlation with lipid profile parameters.

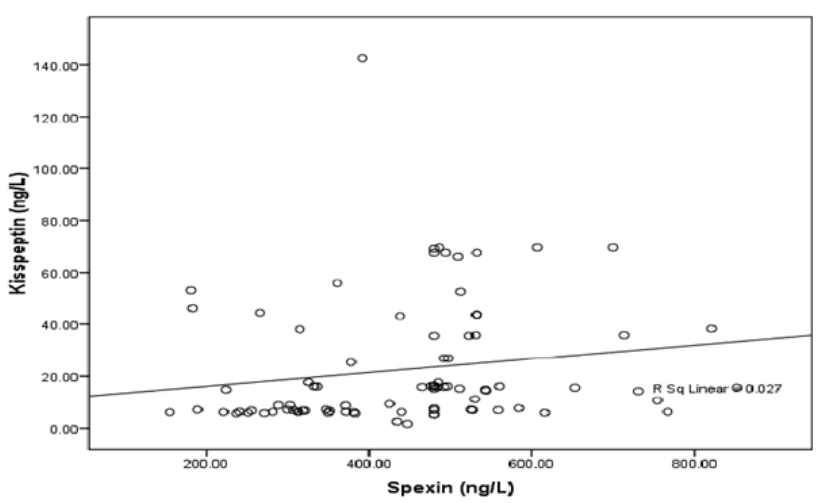

Figure-II: Scatter plot and correlation analysis between $\mathrm{KP}$ and SPX in subjects with normal and altered sperm parameters.

Table-II: Correlation of spexin and kisspeptin with lipid profile.

\begin{tabular}{ccccc}
\hline Study Variables & \multicolumn{2}{c}{ KP(ng/L) } & \multicolumn{2}{c}{ SPX (ng/L) } \\
& r-value & p-value & r-value & p-value \\
\hline Age (Years) & 0.189 & 0.077 & 0.164 & 0.124 \\
$\begin{array}{c}\text { Body mass index } \\
\text { (kg/m2) }\end{array}$ & 0.007 & 0.947 & -0.057 & 0.600 \\
Body Fat (\%) & 0.091 & 0.394 & 0.005 & 0.963 \\
\hline Kisspeptin (ng/L) & - & - & 0.332 & $<0.001$ \\
\hline $\begin{array}{c}\text { Spexin (ng/L) } \\
\text { Cholesterol (mg/dl) }\end{array}$ & -0.332 & $<0.001$ & - & - \\
\hline Triglycerides (mg/ & 0.023 & 0.832 & -0.028 & 0.796
\end{tabular}

dl)

\begin{tabular}{ccccc}
$\begin{array}{c}\text { Low Density } \\
\text { Lipoprotein C3 } \\
(\mathrm{mg} / \mathrm{dl})\end{array}$ & 0.039 & 0.719 & 0.041 & 0.705 \\
\hline $\begin{array}{c}\text { High density lipid } \\
\text { protein C4 (mg/dl) }\end{array}$ & -0.154 & 0.152 & 0.160 & 0.135 \\
\hline \multicolumn{5}{c}{ DISCUSSION } \\
\hline
\end{tabular}

The results obtained in the present study show that serum concentrations of SPX and KPwere significantly less in 'altered sperm parameters' males. This demonstrates that both peptides may be involved in the pathogenesis of infertility. The findings for KP corroborate the findings of Rehman et al., who showed that within a Pakistani population sample, 'altered sperm parameters' males had significantly lower levels of KP, and as such, had significantly lesser concentrations of FSH and LH $(\mathrm{p}<0.001)^{[3]}$. Furthermore, our results for KP are in accordance with a study from Iran ${ }^{[18]}$. Extensive literature can already be found on KP and its role in fertility, whereby it is now unanimously accepted that KP is responsible for driving fertility through the release of GnRH and subsequently LH and FSH ${ }^{[19]}$. Following successful trials of KP injection now being approved for use in treating infertility, previous research has shown that injections of KP near GnRH producing neurons causes a rise in LH production ${ }^{[20]}$. No significant correlation between KP and any of the lipid profile parameters (LDL, HDL, and triglyceride and cholesterol) or between KP and BMI were observed. 
These results are different from the results obtained by Kolodziejski et al., who observed a statistically significant negative correlation between KP, SPX and BMI ${ }^{[4]}$. Furthermore, previous studies have indicated that KP may be a key link between the nutritional status of an individual and their fertility ${ }^{[21]}$. It has long been seen that energy balance and nutritional status are important predictors for successful reproduction since in order to reproduce, the organism must have adequate nutritional resources. Previous literature has supported the concept that in situations of negative energy balance (when the energy required by metabolism is greater than the food intake), hypothalamic KP levels are reduced in mice and rats, either in the whole hypothalamus or in certain nuclei, which then inhibits GnRH production ${ }^{[20]}$. On the other end of the spectrum in positive energy balance leading to obesity, the data is relatively sparse. However, Quennnell et al., documented a diet-induced obesity (DIO) mouse model, obese female DBA/2J mice had lower levels of Kiss1 mRNA in their later oventral periventricular and arcuate nuclei in the hypothalamus, along with lower numbers of KP producing neurons compared to chow fed mice ${ }^{[22]}$. The fact that no significant correlation between KP and BMI was unable to be found in our study indicates that further studies with a larger sample size need to be conducted within a Pakistani setting before making a conclusion. With regards to SPX, as mentioned previously, there is a paucity of information. No similar study for SPX has been carried out in humans. The results from our study showed 'altered sperm parameters' males had a lower level of SPX as compared to fertile males. Unlike KP, SPX injections have not been seen to be effective in modifying FSH or LH mRNA in the anterior pituitary gland in grouper fish ${ }^{[23]}$.

Furthermore, in the DIO mice model, with increased triglyceride levels, it was found that SPX treatment could reverse the elevated total lipid. The exact mechanisms of this interaction remain largely unclear; however, SPX is thought to inhibit the long chain fatty acids accumulation by adipocytes and play a part in weight loss in rodents (possibly through the reducing intake of calories and increasing lipid oxidation) ${ }^{[10]}$.

The impact of obesity on a number of clinical conditions and amplification of cancerous conditions, especially prostatic cancer, has been observed in our country ${ }^{[24]}$. A study was done by Kumar et al. in which a significant positive correlation existed between SPX and KP ${ }^{[24]}$. Our results were supported by previous studies that showed both KP and SPX are lower in 'altered sperm parameters' males and higher in male subjects with 'normal sperm parameters'. On the other hand, this conflicts with the notion that KP stimulates LH release, and SPX inhibits LH release, as mentioned above, hence further research needs to be conducted on this front.

\section{LIMITATIONS}

The limitations of this study include the small sample size and the fact that due to budgetary constraints, certain parameters, including the levels of adipokines (adiponectin and resistin) and insulin resistance parameters could not be measured.

\section{CONCLUSION}

Spexin and kisseptin levels were observed to be higher in male subjects with normal sperm parameters and had a significant positive correlation with each other. There was no impact of spexin on lipid profile and obesity, SPX has no impact on lipid profile and obesity. Further research needs to be conducted to assess the correlation of these peptides with lipid profile on a larger sample size to obtain a strongly significant correlation.

\section{ACKNOWLEDGEMENT:}

We would like to acknowledge our vendor Shariq, who provided us with the ELISA kits. This project was funded by the research module funds provided by the Department of Biological and Biomedical Sciences, Aga Khan University. CONFLICT OF INTEREST: None.

GRANTSUPPORT\& FINANCIAL DISCLOSURE:None.

\section{REFERENCES:}

1. Kim D-K, Yun S, Son GH, Hwang J-I, Park CR, Kim JI, et al. Coevolution of the Spexin/Galanin/Kisspeptin Family: Spexin activates galanin receptor type II and III. Endocrinology. 2014;155(5):1864-1873, Doi:10.1210/ en.2013-2106

2. Rehman R, Alam A, Zafar S. Kisspeptin Levels in Infertile Male Subjects with Abnormal Sperm Parameters, The Journal of the Pakistan Medical Association. 2019;69(8): 1227-1228.

3. Rehman R, Fatima SS, Alam F, Ashraf M, Zafar S. Kisspeptin and Attributes of Infertile males and Females: A Cross-Sectional Study in a Subset of Pakistani Population. Andrologia. 2019;51(9): e13370, Doi:10.1111/and.13370

4. Kołodziejski P, Pruszyńska-Oszmałek E, Korek E, Sassek M, Szczepankiewicz D, Kaczmarek P, et al. Serum Levels of Spexin and Kisspeptin Negatively Correlate with Obesity and Insulin Resistance in Women. Physiological Research. 2018;67(1):45-56. Doi: 10.33549/physiolres.933467

5. Zhou Q, Chen H, Yang S, Li Y, Wang B, Chen Y, et al. High-Fat Diet Decreases the Expression of Kiss1 mRNA and Kisspeptin in the Ovary, and Increases Ovulatory Dysfunction in Postpubertal Female Rats. Reproductive Biology and Endocrinology. 2014;12(1):1-11.

6. Rehman R, Jamil Z, Khalid A, Fatima SS. Cross Talk Between Serum Kisspeptin-Leptin During Assisted Reproduction Techniques. Pakistan Journal of Medical Sciences. 2018;34(2):342-346, Doi: 10.12669/ pjms.342.14078

7. PałaszA, Suszka-ŚwitekA, KaśkoszA, Plewka D, Bogus K, Filipczyk Ł, et al. Spexin-Expressing Neurons in The Magnocellular Nuclei of the Human Hypothalamus. Journal of Chemical Neuroanatomy. 2021; 111:101883. Doi: 10.1016/j.jchemneu.2020.101883

8. Ma A, Bai J, He M, Wong AO. Spexin as a Neuroendocrine Signal with Emerging Functions. General and Comparative Endocrinology. 2018; 265:90-96. Doi: 10.1016/j.ygcen.2018.01.015. 
9. Lin C-y, Huang T, Zhao L, Zhong LL, Lam WC, Fan B-m, et al. Circulating spexin levels negatively correlate with age, BMI, fasting glucose, and triglycerides in healthy adult women. Journal of the Endocrine Society. 2018;2(5):409-419. Doi: 10.1210/js.2018-00020.

10. Walewski JL, Ge F, Gagner M, Inabnet WB, Pomp A, Branch $\mathrm{AD}$, et al. Adipocyte accumulation of longchain fatty acids in obesity is multifactorial, resulting from increased fatty acid uptake and decreased activity of genes involved in fat utilization. Obesity Surgery. 2010;20(1):93-107. Doi: 10.1007/s11695-009-0002-9

11. Gu L, Ma Y, Gu M, Zhang Y, Yan S, Li N, et al. Spexin peptide is expressed in human endocrine and epithelial tissues and reduced after glucose load in type 2 diabetes. Peptides. 2015; 71:232-239. Doi: 10.1016/j. peptides.2015.07.018.

12. Behrooz M, Vaghef-Mehrabany E, Ostadrahimi A. Different spexin level in obese vs normal weight children and its relationship with obesity related risk factors. Nutrition, Metabolism and Cardiovascular Diseases. 2020;30(4):674-682. Doi: 10.1016/j. numecd.2019.11.008.

13. Lv S-Y, Zhou Y-C, Zhang X-M, Chen W-D, Wang Y-D. Emerging roles of NPQ/spexin in physiology and pathology. Frontiers in Pharmacology. 2019; 10:457. Doi:10.3389/fphar.2019.00457

14. Zheng B, Li S, Liu Y, Li Y, Chen H, Tang H, et al. Spexin suppress food intake in zebrafish: Evidence from gene knockout study. Scientific Reports. 2017;7(1):1-9. Doi:10.1038/s41598-017-15138-6

15. Cooper TG, Noonan E, von Eckardstein S, Auger J, Baker HW et al. World Health Organization reference values for human semen characteristics. Human Reproduction Update. 2010; 16 (3): 231-245. Doi: 10.1093/humupd/dmp048

16. Appropriate body-mass index for Asian populations and its implications for policy and intervention strategies. Lancet (London, England). 2004;363(9403):157-163.

17. Zahid N, Saleem S, Azam I, Moatter T. Association of obesity with infertility among Pakistani men: a case control study. Open Journal of Epidemiology. 2015;5(03):204-215. Doi:10.4236/ojepi.2015.53025.

18. Ramzan MH, Ramzan M, Ramzan F, Wahab F, Jillani M, Khan MA, et al. Insight into the serum kisspeptin levels in infertile males. Archives of Iranian Medicine. 2015;18(1):12-17.

19. De Bond J-A, Smith JT. Kisspeptin and energy balance in reproduction. Reproduction. 2014;147(3): R53-R63. Doi:10.1530/REP-13-0509

20. Patterson M, Murphy K, Thompson E, Patel S, Ghatei M, Bloom S. Administration of kisspeptin-54 into discrete regions of the hypothalamus potently increases plasma luteinising hormone and testosterone in male adult rats. Journal of Neuroendocrinology. 2006;18(5):349-354. Doi: $10.1111 / \mathrm{j} .1365-2826.2006 .01420 . x$
21. Castellano J, Navarro V, Fernandez-Fernandez R, Nogueiras R, Tovar S, Roa J, et al. Changes in hypothalamic KiSS-1 system and restoration of pubertal activation of the reproductive axis by kisspeptin in undernutrition. Endocrinology. 2005;146(9):39173925. Doi:10.1210/en.2005-0337.

22. Quennell JH, Howell CS, Roa J, Augustine RA, Grattan DR, Anderson GM. Leptin deficiency and diet-induced obesity reduce hypothalamic kisspeptin expression in mice. Endocrinology. 2011;152(4):1541-1550. Doi: 10.1210/en.2010-1100

23. Li S, Liu Q, Xiao L, Chen H, Li G, Zhang Y, et al. Molecular cloning and functional characterization of spexin in orange-spotted grouper (Epinephelus coioides). Comparative Biochemistry and Physiology Part B: Biochemistry and Molecular Biology. 2016; 196:85-91. Doi: 10.1016/j.cbpb.2016.02.009

24. Bashir MN, Malik MA, Ahmad MR. A case-control study on prostate cancer in rural population of Pakistan. Journal of University Medical \& Dental College. 2015;6(3):56-60. Doi: 10.7314/apjcp.2015.16.6.2375

25. Kumar S, Hossain M, Javed A, Kullo I, Balagopal PB. Relationship of circulating spexin with markers of cardiovascular disease: A pilot study in adolescents with obesity. Pediatric Obesity. 2018;13(6):374-380. Doi: 10.1111/ijpo. 12249

Author's Contribution:

Mohummad Hassan Raza: Idea conception, and manuscript writing.

Mussarat Ashraf: Data collection, and manuscript review. Sher Khan: Correction and revision

Nida Farooqui: Data analysis.

Arfa Azhar: Data compilation, proof reading and critical analysis.

Rehana Rehman: Conception, design and final review of the manuscript.

Submitted for Publication:19-03-2021 Accepted after revision: 23-06-2021 